\title{
Método analítico para a determinação de meloxicam em plasma humano por cromatografia líquida de alta eficiência (CLAE)
}

\author{
Valentina Porta*, Humberto Gomes Ferraz, Tatiane Maria de Lima Souza, Eunice Kazue Kano, \\ Cristina Helena dosReisSerra
}

Departamento de Farmácia, Faculdade de Ciências Farmacêuticas, Universidade de São Paulo

\footnotetext{
*Correspondência:

V. Porta

Departamento de Farmácia, FCF-USP

Av. Lineu Prestes, 580 Bloco 15

Cidade Universitária "Armando Salles

Oliveira"

055089-100 - São Paulo - Brasil

E-mail:vporta@usp.br
}

Desenvolveu-se e validou-se método analitico simples, rápido e específico para quantificação de meloxicam (inibidor da COX-2) em plasma humano através da cromatografia líquida de alta eficiência, para aplicação em estudos de bioequivalência. Piroxicam foi utilizado como padrão interno. Empregou-se cromatografia em fase reversa com coluna modelo Synergi RP$\operatorname{MAX}(150 \times 4,6 \mathrm{~mm})$, à temperatura de $30{ }^{\circ} \mathrm{C}$ e fase móvel constituida por mistura de acetonitrila e tampão fosfato 0,025 mol/L pH 4,5 (40:60, v/v), a um fluxo de 1,0 mL/min. Os analitos foram detectados por UV a $364 \mathrm{~nm}$. As amostras de plasma foram acidificadas com ácido clorídrico $1 \mathrm{~mol} / \mathrm{L}$, extraídas utilizando-se éter terc-butil metílico e, após filtração e secagem, o resíduo foi reconstituido em $250 \mathrm{~mL}$ de fase móvel para injeção em CLAE. Os tempos de retenção para meloxicam (padrão) e piroxicam (padrão interno) foram 3,35 e 4,19 minutos, respectivamente. Este método apresentou linearidade na faixa de concentração entre 50-4000 $\mathrm{ng} / \mathrm{mL}\left(R^{2}=0,9995\right)$, com limite de quantificação inferior de $50 \mathrm{ng} / \mathrm{mL}$ e exatidão de $114 \%$. O método analítico desenvolvido neste trabalho demonstrou especificidade, linearidade, precisão e exatidão adequadas, permitindo sua aplicação em ensaios de bioequivalência.
Unitermos

- Meloxicam/determinação

- Plasma humano

- Cromatografia líquida de alta eficiência (CLAE)

\section{INTRODUÇÃO}

Meloxicam, um derivado enolcarboxamídico relacionado com os oxicanos, quimicamente denominado 4hidroxi-2-metil- $\mathrm{N}$-(5-metil-2-tiazolil)-2H-1,2-benzotiazina3-carboxamida 1,1-dioxano, possui alta atividade inibitória e seletiva sobre a cicloxigenase (COX-2), o que lhe confere considerável atividade antiinflamatória e analgésica, além de excelente tolerância com reduzidos efeitos colaterais sobre a mucosa gastrintestinal (Merck, 1996; Korolkovas, 1999; Vade-Mécum, 1999).

O fármaco é bem absorvido por via oral, apresentando biodisponibilidade de cerca de $89 \%$ e atingindo a concentração plasmática máxima após 5 a 6 horas. Sua meiavida de eliminação é de aproximadamente 20 horas (Türck et al., 1997; Korolkovas, 1999). 
Diversos métodos para uso em estudos farmacocinéticos, notadamente envolvendo a cromatografia líquida de alta eficiência (CLAE), têm sido propostos para quantificação do meloxicam em líquidos biológicos (Türck et al, 1997; Radhofer-Welte, Dittrich, 1998; Velpandian et al., 2000; Elbary et al., 2001). Tais métodos apresentam algumas desvantagens que dificultam sua aplicação em ensaios de bioequivalência, devido à baixa resolução cromatográfica, procedimentos complexos e demorados de extração ou em razão do uso de solventes demasiadamente tóxicos.

Velpandian et al. (2000) descrevem método que emprega clorofórmio como solvente extrator para a obtenção de valores de recuperação próximos a $100 \%$ para o fármaco e o padrão interno (piroxicam). Entretanto, tanto este método, quanto o proposto por Elbary et al. (2001) apresentam faixas de linearidade inadequadas à sua aplicação em ensaios de biodisponibilidade e bioequivalência, respectivamente de 520 a $5200 \mathrm{ng} / \mathrm{mL}$ e de 40 a $800 \mathrm{ng} / \mathrm{mL}$.

Assim, o objetivo do presente trabalho foi, baseando-se nos trabalhos citados, desenvolver e validar um método analítico por CLAE para quantificação do fármaco em plasma humano que apresentasse adequada sensibilidade e facilidade com relação aos procedimentos de extração, para uso em estudos de bioequivalência envolvendo formulações de meloxicam.

\section{MATERIAL E MÉTODOS}

\section{Reagentes}

Utilizou-se padrão secundário de meloxicam $(99,5 \%)$ e padrão secundário de piroxicam (padrão interno, 99,6\%), doados por Cadila Healthcare Limited e Galena, respectivamente. Os solventes utilizados foram de grau CLAE, fornecidos pela Merck S.A. O tampão fosfato $(0,025 \mathrm{~mol} / \mathrm{L})$ empregado na fase móvel foi obtido a partir de reagente Merck S.A., e de água ultra-pura obtida em equipamento Milli-Q (Millipore). O ácido clorídrico 1,0 mol/L utilizado na extração das amostras de plasma foi fornecido pela Merck S.A.. O plasma branco de referência para preparo das amostras adicionadas de padrão e de controle de qualidade foi doado pela Fundação PróSangue, Hemocentro de São Paulo. Os plasmas branco normal, lipêmico e hemolisado foram obtidos de voluntários sadios sem exposição ao meloxicam.

\section{Equipamento}

A pesagem dos padrões para preparo de soluções estoque, solução de padrão interno e amostras de plasma foi feita em balança analítica Mettler, modelo AB204-S. As diluições foram realizadas utIlizando-se pipetas e micropipetas automáticas das marcas Brand e Eppendorf e vidraria volumétrica certificada.

Para o procedimento de extração das amostras de plasma empregaram-se pipetas e micropipetas automáticas das marcas Brand e Eppendorf, agitador de tubos tipo vórtex da marca Phoenix, evaporador RapidVap ${ }^{\circledR}$ Labconco, seringa de vidro e unidades filtrantes HV Millex com membrana Durapore ${ }^{\circledR}$ de $13 \mathrm{~mm}$ de diâmetro e poro de $0,45 \mathrm{~mm}$.

A análise cromatográfica foi executada em equipamento de cromatografia líquida de alta eficiência MerckHitachi ${ }^{\circledR}$ composto por uma bomba modelo L-7100, desgaseificador modelo L-7612, injetor automático de amostras modelo L-7200, detector UV-visível modelo L7400 , forno de coluna modelo L-7300 e unidade de controle modelo LD-7000.

\section{Soluções estoque, solução de padrão interno e amostras de plasma padrão e de controle de qualidade}

A solução estoque de meloxicam para preparo de amostras de plasma padrão (Sol. A) foi obtida dissolvendo-se $100 \mathrm{mg}$ de meloxicam em $100 \mathrm{~mL}$ de metanol (concentração $=1000 \mu \mathrm{g} / \mathrm{mL}$ ). As amostras de plasma padrão nas concentrações de 50, 200, 500, 1000, 1500 e 3000 ng/mL foram obtidas por meio de diluições sucessivas da Sol. A em plasma branco.

As amostras de plasma para o controle de qualidade nas concentrações de 150, 1000 e $2500 \mathrm{ng} / \mathrm{mL}$ foram obtidas por meio de diluições sucessivas da Sol. B (preparada da mesma forma que a Sol. A) em plasma branco.

A solução de padrão interno foi obtida dissolvendose $50 \mathrm{mg}$ de piroxicam em $100 \mathrm{~mL}$ de metanol (concentração $=500 \mu \mathrm{g} / \mathrm{mL}$ ).

\section{Preparação das amostras de plasma para quantificação de meloxicam}

A extração de meloxicam das amostras de plasma foi realizada com éter terc-butil-metílico em meio ácido. Adicionaram-se $0,5 \mathrm{~mL}$ de plasma e $200 \mu \mathrm{l}$ de solução de ácido clorídrico $1,0 \mathrm{~mol} / \mathrm{L}$ a tubos contendo $25 \mu \mathrm{L}$ de solução de padrão interno (piroxicam) em metanol na concentração de $10,0 \mu \mathrm{g} / \mathrm{mL}$, homogeneizando-se em agitador de tubos por 30 segundos. A seguir, extraiu-se o plasma com $3,0 \mathrm{~mL}$ de éter terc-butil-metil durante 60 segundos sob agitação vigorosa. Após congelamento da amostra, para diminuir o risco de contaminação da fase orgânica pela fase aquosa, o extrato orgânico foi filtrado em unidade HV 
Millex com membrana Durapore ${ }^{\circledR}$ de $13 \mathrm{~mm}$ de diâmetro e poro de $0,45 \mu \mathrm{m}$. O filtrado foi transferido para tubo cônico onde efetuou-se secagem em equipamento RapidVap ${ }^{\circledR}$ Labconco à temperatura de $40^{\circ} \mathrm{C}$, vácuo de $300 \mathrm{mBar}$, a velocidade de rotação de $25 \mathrm{rpm}$. Reconstituiu-se o resíduo em $250 \mu \mathrm{L}$ de fase móvel para análise por CLAE.

\section{Condições cromatográficas para determinação de meloxicam em amostras de plasma}

Empregou-se para separação coluna de marca Phenomenex ${ }^{\circledR}$, modelo Synergi ${ }^{\circledR}$ RP-MAX, de 15,0 cm de comprimento e 4,6 mm de diâmetro interno, contendo partículas de $4 \mu \mathrm{m}$. O comprimento de onda utilizado para detecção do analito foi $364 \mathrm{~nm}$ e a análise foi realizada à temperatura de $30^{\circ} \mathrm{C}$.

A fase móvel foi constituída por mistura de acetonitrila e tampão fosfato $0,025 \mathrm{~mol} / \mathrm{L} \mathrm{pH} 4,5$ na proporção de 40:60 (v/v), filtrada em membrana NylonPhenomex ${ }^{\circledR}$ de $47 \mathrm{~mm}$ de diâmetro e poro de $0,2 \mathrm{~mm}$, desgaseificada em banho ultrassônico e introduzida no sistema cromatográfico a um fluxo de $1,0 \mathrm{~mL} / \mathrm{min}$.

\section{Validação do método para quantificação de meloxicam em plasma}

A validação do método analítico desenvolvido para quantificação de meloxicam em plasma foi realizada por meio da determinação dos parâmetros de especificidade, linearidade, recuperação, limite de quantificação, precisão, exatidão e estabilidade (Brasil, 2003).

\section{Especificidade}

Tal parâmetro foi investigado pela análise de seis amostras de plasma "branco" sendo uma amostra de plasma lipêmico, uma amostra de plasma hemolisado e quatro amostras de plasma normal, para verificação da existência de interferência de componentes endógenos do plasma na análise do fármaco e do padrão interno.

\section{Linearidade}

Foi estabelecida utilizando-se seis amostras de plasma adicionado de padrão de meloxicam em seis concentrações diferentes $(50,200,500,1000,1500$ e $3000 \mathrm{ng} / \mathrm{mL})$ em sextuplicatas. Estabeleceu-se correlação linear entre concentração, considerada variável independente (x), e relação entre as áreas dos picos cromatográficos do fármaco e do padrão interno, considerada variável dependente (y). Os parâmetros da correlação foram estimados pelo método dos mínimos quadrados.

\section{Recuperação}

Foi determinada comparando-se resultados de análises de amostras de plasma adicionados de padrão submetidas ao processo de extração a resultados de análises de amostras de meloxicam em metanol não submetidas a esse processo, em três diferentes concentrações $(150,1000$ e $2500 \mathrm{ng} / \mathrm{mL}$ ) e cinco repetições.

\section{Limite de quantificação}

O limite de quantificação foi determinado utilizandose amostras de plasma de controle de qualidade em cinco repetições. A precisão deve estar entre $\pm 20 \%$ do valor nominal da concentração, com coeficiente de variação (precisão) de, no máximo, $20 \%$.

\section{Precisão}

Esse parâmetro foi determinado pela análise de amostras de plasma de controle de qualidade em três diferentes concentrações $(150,1000$ e $2500 \mathrm{ng} / \mathrm{mL})$ e cinco repetições (precisão intra-dia $=$ análises realizadas no mesmo dia; precisão inter-dia = análises realizadas em dias diferentes).

\section{Exatidão}

O referido parâmetro foi determinado pela análise de amostras de plasma de controle de qualidade em três diferentes concentrações $(150,1000$ e $2500 \mathrm{ng} / \mathrm{mL})$ e cinco repetições (exatidão intra-dia $=$ análises realizadas no mesmo dia; exatidão inter-dia $=$ análises realizadas em dias diferentes).

\section{Estabilidade}

Determinou-se a estabilidade do meloxicam em amostras de plasma mantidas a $-20^{\circ} \mathrm{C}$ e submetidas a três ciclos de congelamento e descongelamento, além da estabilidade de curta duração (no tempo e condições de análise); estabilidade das soluções estoque e estabilidade de longa duração (Brasil, 2003).

\section{Quantificação de meloxicam em amostras de plasma de voluntário}

Administrou-se comprimido contendo $15 \mathrm{mg}$ de meloxicam a um voluntário sadio, mantido em jejum por oito horas antes e quatro horas após a administração do medicamento, o qual foi ingerido com auxílio de $200 \mathrm{~mL}$ de água. Coletaram-se amostras de sangue em tubo heparinizado nos tempos $0,1,2,3,4,5,6,7,8,12,24,48,72$ e 96 horas após a administração. O sangue foi centrifugado por 10 minutos a $3000 \mathrm{rpm}$ e o plasma obtido foi congelado e mantido a $20{ }^{\circ} \mathrm{C}$ até a análise para quantificação do fármaco. 
As amostras de plasma do voluntário foram analisadas paralelamente à curva de calibração formada por amostras de plasma adicionado de padrão em seis concentrações $(50,200,500,1000,1500$ e $3000 \mathrm{ng} / \mathrm{mL})$ e a amostras de plasma de controle de qualidade em três diferentes concentrações (baixa: $150 \mathrm{ng} / \mathrm{mL}$; média: $1000 \mathrm{ng} / \mathrm{mL}$ e alta: $2500 \mathrm{ng} / \mathrm{mL}$ ) em triplicata.

\section{RESULTADOS}

O método desenvolvido demonstrou-se específico para meloxicam com piroxicam (p. i.) e, obtendo-se boa se- paração deste com piroxicam entre si e dos componentes do plasma, com tempos de retenção de 3,35 e 4,19 minutos, respectivamente (Figura 1).

O método mostrou-se linear entre as concentrações de 50 a $4500 \mathrm{ng} / \mathrm{mL}$ (Figura 2). A precisão variou entre 1,65 e 4,19\% para amostras analisadas no mesmo dia (intra-dia) e entre 3,16 e 7,94\% para amostras analisadas em dias diferentes (inter-dias). A exatidão variou entre 101 e $112 \%$ para análises intra-dia e entre 98 e $106 \%$ para análises inter-dias (Tabela I).

O limite de quantificação do método foi de $50 \mathrm{ng} / \mathrm{mL}$, com precisão de $2,35 \%$ e exatidão de $114 \%$. A recupera-

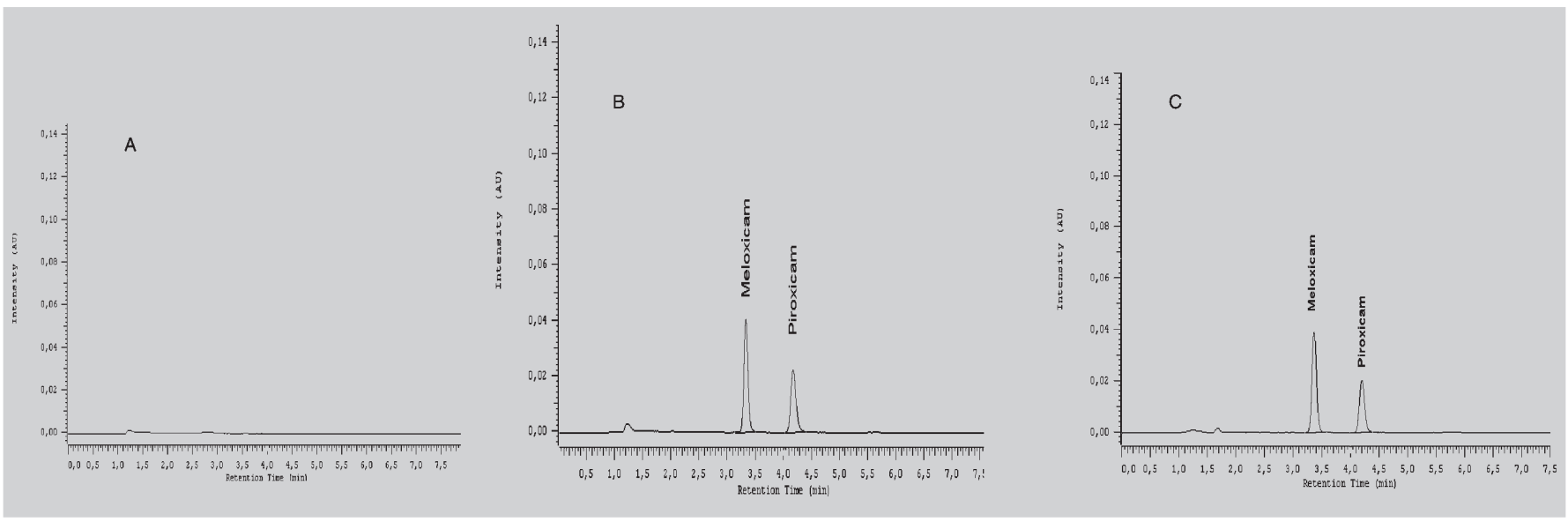

FIGURA 1 - Cromatogramas referentes às amostras de: A - plasma branco normal obtido de voluntário sadio sem administração do meloxicam; B plasma branco adicionado de e padrão de meloxicam $(2,0 \mu \mathrm{g} / \mathrm{mL})$ e de padrão interno ( $50 \mu \mathrm{L}$ de solução de piroxicam em metanol a $10 \mu \mathrm{g} / \mathrm{mL}) ; C$ - plasma obtido de voluntário após administração de $15 \mathrm{mg}$ de meloxicam adicionado de padrão interno $(50 \mu \mathrm{L}$ de solução de piroxicam em metanol a $10 \mu \mathrm{g} / \mathrm{mL})$.

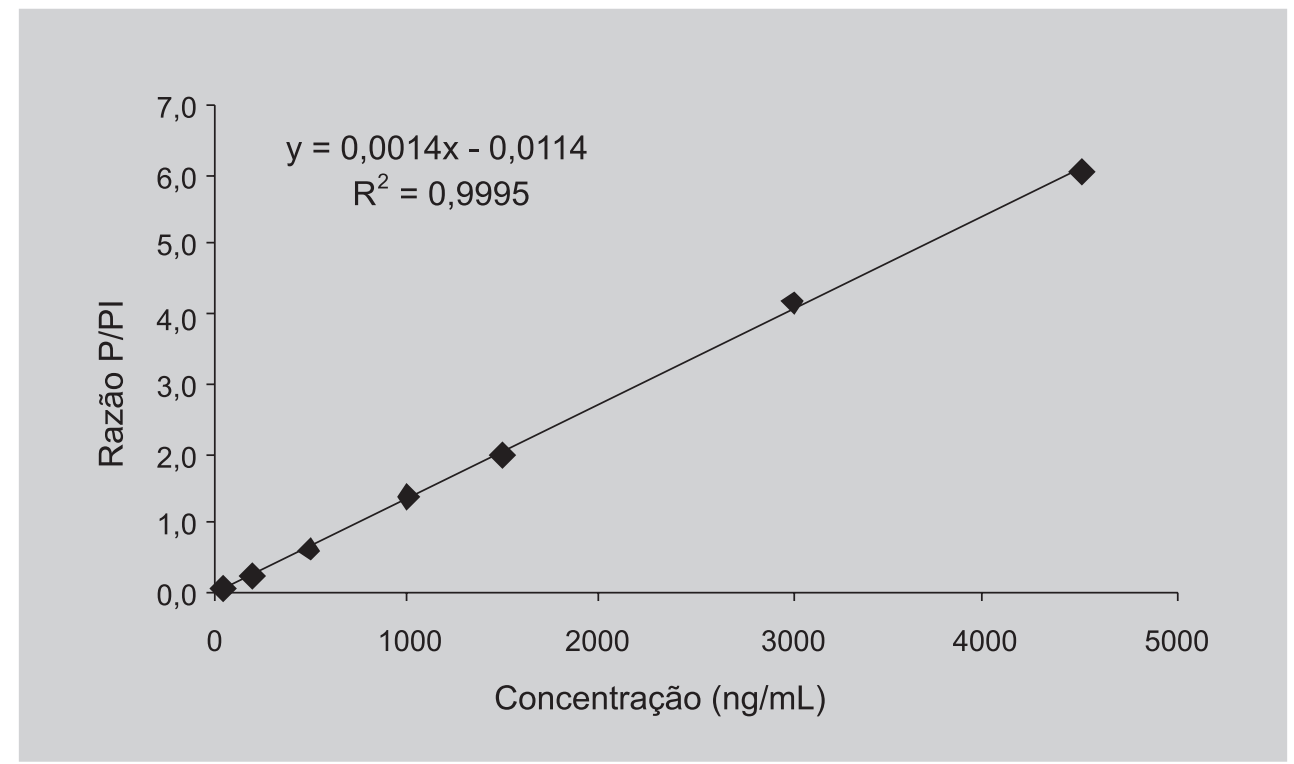

FIGURA 2 - Curva de calibração do método para quantificação de meloxicam em plasma por CLAE na faixa de concentração de 50 a $4500 \mathrm{ng} / \mathrm{mL}$. 
TABELA I - Precisão e exatidão intra-dia e inter-dias referente ao método analítico para quantificação de meloxicam em plasma por cromatografia líquida de alta eficiência. Cada valor representa a média de cinco determinações

\begin{tabular}{lcccc}
\hline $\begin{array}{l}\text { Concentração } \\
(\mathbf{n g} / \mathbf{m L})\end{array}$ & Intra-dia & Precisão (\%) & \multicolumn{2}{c}{ Exatidão (\%) } \\
\hline 150 & 4,19 & Inter-dias & Intra-dia & Inter-dias \\
1000 & 3,09 & 7,16 & 101 & 1060 \\
2500 & 1,65 & 7,94 & 106 & 99 \\
\hline
\end{tabular}

ção média da extração foi de $107 \%$ para meloxicam e $93 \%$ para piroxicam, com precisão e exatidão dentro dos limites especificados (Tabela II).

As amostras de plasma mantiveram-se estáveis a $-20^{\circ} \mathrm{C}$ e a três ciclos de congelamento e descongelamento, conforme apresentado na Tabela III.

A estabilidade de curta duração, representada pela estabilidade no tempo e condições de análise, foi determina-

TABELA II - Recuperação média da extração das amostras de plasma para meloxicam (padrão) e piroxicam (padrão interno). Cada valor representa a média de seis determinações. $\mathrm{CV}=$ coeficiente de variação

\begin{tabular}{lcc}
\hline $\begin{array}{l}\text { Concentração } \\
\text { (ng/mL) }\end{array}$ & $\begin{array}{l}\text { Recuperação (\%) } \\
\text { Padrão } \\
\text { (CV\%) }\end{array}$ & $\begin{array}{c}\text { Padrão interno } \\
\text { (CV\%) }\end{array}$ \\
\hline 150 & $114(3,32)$ & $99(4,35)$ \\
1000 & $104(2,33)$ & $90(3,00)$ \\
2500 & $104(1,39)$ & $89(5,55)$ \\
Média & 107 & 93 \\
\hline
\end{tabular}

da utilizando-se amostras de plasma de controle de qualidade submetidas ao mesmo tratamento que as amostras de plasma de voluntários a serem analisadas, ou seja, descongelamento natural à temperatura ambiente e preparação conforme método validado previamente descrito. Esta estabilidade contemplou o período de espera máximo no injetor automático até a introdução da amostra no sistema cromatográfico (24 horas). As mesmas amostras também foram analisadas após período de 48 horas (Tabela IV).

A estabilidade das soluções estoque de padrão e padrão interno foi determinada pela análise diária das mesmas. A variabilidade apresentada foi de $6,0 \% \mathrm{e}$ $10,18 \%$ para o meloxicam e piroxicam, respectivamente.

A estabilidade das amostras de plasma de controle de qualidade foi acompanhada pela comparação destas com uma curva de calibração preparada no dia de análise. As amostras de plasma de controle de qualidade foram preparadas na sua totalidade antes do início das análises das amostras de plasma do voluntário. As amostras de padrões da curva de calibração, amostras de plasma branco, amostras de plasma de controle de qualidade e amostras de plasma de voluntários que receberam meloxicam

TABELA III - Estabilidade de meloxicam em amostras de plasma mantidas à temperatura de $-20^{\circ} \mathrm{C}$ e submetidas a três ciclos de congelamento e descongelamento. Cada resultado representa a média de três determinações

\begin{tabular}{lcccccc}
\hline $\begin{array}{l}\text { Concentração } \\
\text { (ng/mL) }\end{array}$ & \multicolumn{2}{c}{ Ciclo 1 } & \multicolumn{2}{c}{ Ciclo 2 } & \multicolumn{2}{c}{ Ciclo 3 } \\
\hline 150 & Precisão(\%) & Exatidão(\%) & Precisão(\%) & Exatidão(\%) & Precisão(\%) & Exatidão(\%) \\
1000 & 6,65 & 107 & 3,25 & 111 & 5,34 & 112 \\
2500 & 2,30 & 92 & 0,51 & 114 & 7,98 & 92 \\
\hline
\end{tabular}

TABELA IV - Estabilidade de meloxicam em amostras de plasma no tempo de 24 horas e 48 horas após o preparo. Cada resultado representa a média de três determinações

\begin{tabular}{|c|c|c|c|c|}
\hline \multirow{2}{*}{$\begin{array}{l}\text { Concentração } \\
\text { (ng/mL) }\end{array}$} & \multicolumn{2}{|c|}{24 horas } & \multicolumn{2}{|c|}{48 horas } \\
\hline & Precisão(\%) & Exatidão(\%) & Precisão(\%) & Exatidão(\%) \\
\hline 150 & 3,77 & 95 & 3,14 & 99 \\
\hline 1000 & 8,62 & 110 & 3,54 & 87 \\
\hline 2500 & 5,67 & 113 & 6,11 & 89 \\
\hline
\end{tabular}


mantiveram-se estáveis por 60 dias à temperatura de $20{ }^{\circ} \mathrm{C}$ (Tabela V).

TABELA V - Estabilidade de meloxicam em amostras de plasma mantidas à temperatura de $-20^{\circ} \mathrm{C}$ por 60 dias. Cada resultado representa a média de três determinações

\begin{tabular}{ccc}
\hline $\begin{array}{c}\text { Concentração } \\
(\mathbf{n g} / \mathbf{m L})\end{array}$ & Precisão (\%) & Exatidão (\%) \\
\hline 50 & 2,13 & 111 \\
1000 & 2,79 & 94 \\
2500 & 7,32 & 100 \\
\hline
\end{tabular}

A Figura 3 apresenta a curva de "concentração plasmática versus tempo" obtida após a administração de uma dose única de $15 \mathrm{mg}$ de meloxicam a voluntário sadio.

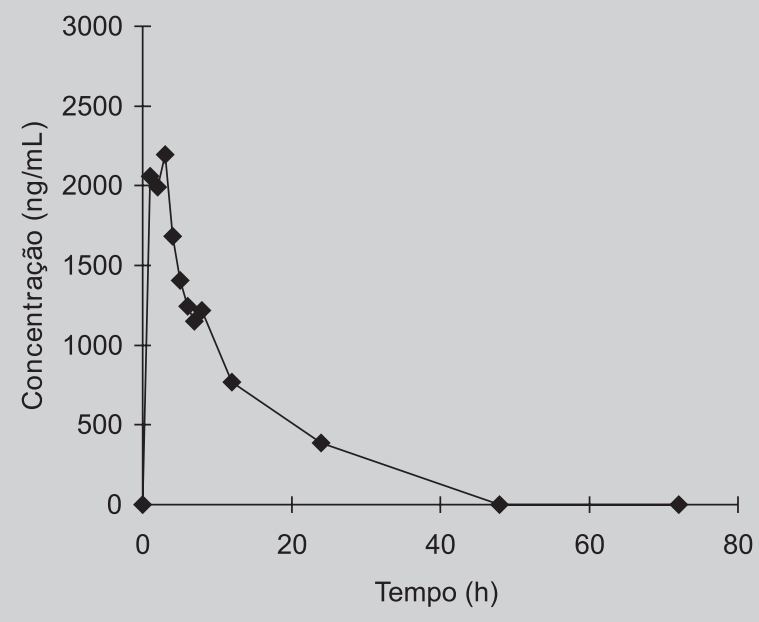

FIGURA 3 - Curva de "concentração plasmática versus tempo" obtida após a administração oral de $15 \mathrm{mg}$ de meloxicam a voluntário sadio

\section{DISCUSSÃO}

Os parâmetros de especificidade, sensibilidade, linearidade, precisão e exatidão obtidos permitem a utilização do método analítico desenvolvido para quantificação de meloxicam em amostras de plasma em ensaios de bioequivalência ou estudos farmacocinéticos envolvendo formulações contendo o referido fármaco. Este método foi utilizado para quantificação do fármaco em amostras de plasma de voluntário, ao qual foi administrado comprimido contendo $15 \mathrm{mg}$ de meloxicam, permitindo a definição da curva "concentração plasmática versus tempo".

$\mathrm{O}$ método mostrou-se específico para o fármaco e para o padrão interno (piroxicam), os quais apresentaram boa separação entre si e dos componentes do plasma, com tempos de retenção de 3,35 e 4,19 respectivamente, permitindo tempo total de análise de cada amostra em torno de 8 minutos. Os picos cromatográficoss obtidos apresentaram-se simétricos e com excelente resolução da linha de base. Os ensaios de bioequivalência geram cerca de 700 amostras de plasma, que devem ser analisadas paralelamente a amostras de plasma padrão e a amostras de plasma de controle de qualidade. Assim, é interessante que os métodos analíticos para estes ensaios apresentem tempo curto de análise, conforme obtido no presente estudo.

O limite de quantificação do presente método (50 ng/mL) mostrou-se adequado à análise de meloxicam nas amostras de plasma do voluntário que recebeu o fármaco. No caso de ensaios de bioequivalência, este parâmetro adquire extrema importância, uma vez que, nestes estudos, o tempo de coleta de amostras deve corresponder a, no mínimo, 3,5 vezes a meia-vida de eliminação do fármaco. Para o meloxicam, que apresenta meiavida de eliminação média de 20 horas, as coletas devem ser realizadas até, no mínimo, 70 horas após a administração do medicamento. No presente estudo, obtiveram-se amostras até 96 horas após a administração do medicamento, tendo sido possível quantificar o fármaco inclusive na última amostra coletada.

Outro parâmetro a ser considerado em métodos analíticos a serem aplicados em ensaios de bioequivalência é a linearidade, já que é necessária a quantificação do fármaco na concentração máxima $\left(\mathrm{C}_{\max }\right)$. No presente trabalho, o valor de $\mathrm{C}_{\text {max }}$ médio obtido foi de $2196,98 \mathrm{ng} / \mathrm{mL}$, dentro da faixa de linearidade estipulada para o método (50 a $4500 \mathrm{ng} / \mathrm{mL}$ ).

As amostras de plasma utilizadas neste trabalho mantiveram-se estáveis por 60 dias à temperatura de $-20{ }^{\circ} \mathrm{C}$, e os extratos orgânicos correspondentes, por 24 horas à temperatura ambiente, após a reconstituição com fase móvel. Esta estabilidade é adequada à manutenção das amostras de plasma de voluntários do momento da coleta até o momento da análise e, também, à manutenção dos extratos orgânicos em auto-injetor até sua efetiva introdução no sistema cromatográfico.

Existem alguns métodos descritos para quantificação de meloxicam em amostras de plasma (Türck et al., 1997; Radhofer-Welte, Dittrich, 1998; Velpandian et al., 2000; Elbary et al., 2001). Türck et al. (1997) utilizam extração em fase sólida para preparação das amostras de plasma, enquanto os outros utilizam extração líquido-líquido, da mesma forma que o método desenvolvido neste trabalho.

Ao contrário de Velpandian et al., no presente estudo optou-se pelo éter terc-butil-metílico, menos tóxico em rela- 
ção ao clorofórmio, como solvente extrator. Desta forma, obteve-se recuperação ao redor de $100 \%$ para o meloxicam e de $90 \%$ para o piroxicam. Segundo Bressolle et al. (1996), valores de recuperação próximos a 100\% são desejáveis, mas valores menores são aceitáveis, desde que reprodutíveis. A utilização do éter terc-butil-metílico não trouxe prejuízo ao desempenho do método, o que pode ser confirmado pela análise dos resultados referentes à validação do mesmo, e apresentou vantagens em relação à segurança.

A linearidade do método proposto neste trabalho (50 a $3500 \mathrm{ng} / \mathrm{mL}$ ) inclui toda a faixa de concentração provável após administração de comprimidos contendo $15 \mathrm{mg}$ de meloxicam, ao contrário do que ocorre com os métodos propostos por Elbary et al. (2001) e Velpandian et al. (2000).

Ainda em comparação ao método de Elbary et al. (2001), foi possível obter, no método aqui proposto, um menor tempo de retenção e, conseqüentemente, menor tempo de análise.

Assim, o presente método apresenta algumas vantagens em relação a métodos previamente propostos, especialmente em relação à segurança do procedimento de extração, linearidade e tempo de análise.

Em comparação aos outros descritos na literatura (Turck, et al., 1997; Radhofer-Welte e Dittrich, 1998), o método desenvolvido mostrou vantagens em relação à sensibilidade ou à maior facilidade nos procedimentos de purificação das amostras.

\section{CONCLUSÕES}

O método analítico desenvolvido para quantificação de meloxicam em amostras de plasma por meio de cromatografia líqüida de alta eficiência (CLAE) apresentou especificidade, sensibilidade, linearidade, precisão e exatidão adequadas que permitem sua utilização em ensaios de bioequivalência ou estudos farmacocinéticos envolvendo o referido fármaco.

\section{ABSTRACT \\ Determination of meloxicam in human plasma by HPLC}

A simple, rapid and specific high-performance liquid cromatographic (HPLC) method was developed and validated to estimate meloxicam (COX-2 inhibitor) levels in human plasma. Piroxicam was used as internal standard. Reversed phase chromatography was conducted using a Synergi RP-MAX (150 x $4.6 \mathrm{~mm})$ column at $30^{\circ} \mathrm{C}$ and a mobile phase of acetonitrile and $0.025 \mathrm{~mol} / \mathrm{L} \mathrm{pH} 4,5$ phosphate buffer $(40: 60, \mathrm{v} / \mathrm{v})$, at a flow rate of $1 \mathrm{~mL} / \mathrm{min}$. Analytes were detected at $364 \mathrm{~nm}$. Plasma samples were acidified with $1 \mathrm{~mol} / \mathrm{L}$ hydrochloric acid and extracted with tert-butyl methyl ether, evaporated to dryness, reconstituted in $250 \mu \mathrm{L}$ of mobile phase and injected in the column. The retention time of meloxicam and piroxicam were 3.35 and 4.19 minutes, respectively. This method showed to be linear in the range of $50-4500 \mathrm{ng} / \mathrm{mL}$ $\left(R^{2}=0.9995\right)$, a LOQ of $50 \mathrm{ng} / \mathrm{mL}$ and accuracy of $114 \%$. The analytical method showed suitable specificity, sensitivity, linearity, precision and accuracy, and can be used in bioequivalence or pharmacokinetics studies involving meloxicam.

UNITERMS: Meloxicam. Piroxicam. Plasma. HPLC

\section{REFERÊNCIAS BIBLIOGRÁFICAS}

BRASIL. Leis, decretos, etc. Resolução no 41, de 28 de abril 2000. Diário Oficial da União, Brasília, 28 de abril de 2000. Seção 1. p. 62-69 [A Agencia Nacional de Vigilância Sanitária aprova o regulamento técnico para medicamentos genéricos]. 2000.

BRASIL. Leis, decretos, etc. Resolução no 899, de 29 de maio de 2003. Diário Oficial da União, Brasília, 02 de junho de 2003. Seção 1. p. 56-59 [A Agência Nacional de Vigilância Sanitária aprova o Guia para validação de métodos analíticos e bioanalíticos]. 2003.

BRESSOLLE, F., BROMET-PETIT, M., AUDRAN, M. Validation of liquid chromatographic and gas chromatographic methods. Application to pharmacokinetics. J. Chromatogr. B, v. 686, p. 3-10, 1996.

CAUSON, R. Validation of chromatographic methods in biomedical analysis. Viewpoint and discussion. $J$. Chromatogr. B, v. 689, p. 175-80, 1997.

ELBARY, A. A., FODA, N., ELKHATEEB, M. Reversed phase liquid chromatographic determination of meloxicam in human plasma and its pharmacokinetic application. Anal. Lett., v. 34, p.1175-1187, 2001.

KOROLKOVAS, A. Dicionário Terapêutico Guanabara. Rio de Janeiro: Guanabara Koogan, 1999. p. 21.6

MERCK index: an encyclopedia of chemicals, drugs and biological. 12. ${ }^{\text {a }}$ ed. New Jersey: Whitehouse Station, 1996. p. 582 
RADHOFER-WELTE, S. DITTRICH, P., Determination of the novel non-steroidal anti-inflammatory drug lornoxicam and its main metabolite in plasma and synovial fluid. $J$. Chromatogr. B, v.707, p151-159, 1998.

TURCK, D. BUSCH, U. HEINZEL, G. NARJES, H. Clinical pharmacokinetics of Meloxicam. Arzneimittelforschung, v.47, p.253-258, 1997.
VELPANDIAN, T., JAISWAL, J., BHARDWAJ, R.K., GUPTA, S.K., Development and validation of a new highperformance liquid chromatographic estimation method of meloxicam in biological samples. J. Chromatogr. $B$, v. 738, p. 431-436, 2000.

Recebido para publicação em 14 de maio de 2004. Aceito para publicação em 12 de abril de 2005.

VADE-MÉCUM. São Paulo: Soriak, 1999, p. 431. 
O arquivo disponível sofreu correções conforme ERRATA publicada no Volume 41 Número 3 da revista. 